\title{
On the Errors of a Thin-Film Mounted Rocketsonde Thermistor
}

\author{
Donald C. Thompson \\ New Zealand Meteorological Service, Wellington
}

7 September 1967

In a recent paper, Ballard (1967) presented values for the temperature errors of a modified rocketsonde, the STS-1, which were considerably smaller than those which the author would have expected on the basis of his recent work on the characteristics of thermistor temperature sensors at high altitudes (Thompson, 1966; Thompson and Keily, 1967). Ballard arrived at his values from theoretical considerations and some experimental results. However, before readers place too much reliance on these figures it should be pointed out that some of the arguments used require qualification.

Ballard's expressions (3.1), (3.4) for the dissipation factors $S_{f}, S_{t m}$ are not exact. Thus, in (3.1) no allowance bas been made for the fact that the effective emissivity of a $0.001-\mathrm{cm}$ clear mylar film is considerably less than unity. Again, in (3.4) no allowance has been made for (a) heat lost to the air directly from the thermistor lead wires, (b) local effects at the point of attachment of the thermistor leads to the film, and (c) radiation from thermistor lead wires.

In discussing the solar radiation error in Section 3a, Ballard states that with an oscillating record the temperature minima may be taken as the environmental temperature. This is a dubious procedure as no allowance is made for radiation reflected from below, or for the possibility of fluctuations in the heat transfer characteristics of the sensor with the changing airflow. The application of a $400 \AA$ aluminum film to the mylar film may increase rather than decrease the solar radiation error. An aluminum film of this thickness will have very little transmission of solar radiation so that practically all the energy not reflected will be absorbed, and passed by thermal conduction to the mylar. Thus, the effective reflectivity of the coated mount will be either $90 \%$ or approximately $100 \times 0.9 \times 0.95^{2}=81 \%$, depending on whether the aluminum or the mylar side faces the radiation. The temperature rise of the film on this assumption would be some 20-40 times Ballard's estiestimate.

Inspection of the equations used in Sections $5 \mathrm{a}, \mathrm{b}, \mathrm{c}$ shows that the results are extremely sensitive to the as- sumptions used. For example, in view of the rapid response of the film to changes in air temperature, Ballard's assumption that $T_{f 2}-T_{f 1}=0$ in Section $5 \mathrm{a}$ does not seem plausible when $T_{e 2}-T_{e 1}=10 \mathrm{C}$. However, if one substitutes a more realistic value of say $6.5 \mathrm{C}$ for $T_{f_{2}}-T_{f_{1}}$, one obtains a negative value for the heat transfer coefficient $h_{\mathrm{l}}$. Similarly, the assumption $T_{f}=T_{\text {o }}$ used in Section $5 \mathrm{c}$ to obtain a lower limit of $5 \mathrm{C}$ for the temperature error is unrealistic, since the film would be subjected to aerodynamic heating of some $12-15 \mathrm{C}$ at $65 \mathrm{~km}$ even if there were no other sources of error. If, as an illustration, one assumes $T_{f}=T_{a}+12 \mathrm{C}$, Eq. (5.9) gives $T-T_{0}=13 \mathrm{C}$. Even this value has limited meaning, however, as Eq. (5.8) does not include the effect of solar radiation on the sensor.

Finally, one would expect aerodynamic heating errors alone to be considerably larger than the errors given in Table 3 for the film-mounted thermistor. The exact recovery factor for the film-mounted thermistor will be intermediate between that for a "post"-mounted thermistor and that for a thin flat plate. A value near 1.25 seems reasonable for the former at $65 \mathrm{~km}$, and 1.0 for the latter, so that with a relative velocity of 167 $\mathrm{m} \mathrm{sec}^{-1}$, an aerodynamic heating error of $12-15 \mathrm{C}$ would be expected at this altitude. In general, the other sources of error result in further heating of the thermistor bead above the recovery temperature, so one would expect the total errors to certainly be larger than $12-15 \mathrm{C}$.

It would thus appear that Ballard's results do not give a reliable indication of the temperature errors of a thin-film mounted thermistor.

\section{REFERENCES}

Ballard, H. N., 1967: The measurement of temperature in the stratosphere and mesosphere. J. Appl. Meteor., 6, 150-163.

Thompson, D. C., 1966: The accuracy of miniature bead thermistors in the measurement of upper air temperatures. Sci. Rept. AFCRL-66-773, Air Force Cambridge Research Laboratories, Bedford, Mass., 264 pp.

- and D. P. Keily, 1967: The accuracy of thermistors in the measurement of upper air temperatures. J. Appl. Meleor., 6, 380-385. 